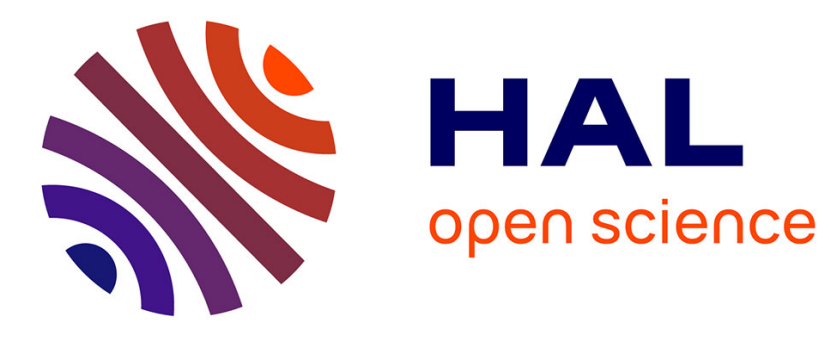

\title{
Dimensioning microwave wireless networks
}

Alvinice Kodjo, Brigitte Jaumard, Napoleão Nepomuceno, Mejdi Kaddour, David Coudert

\section{To cite this version:}

Alvinice Kodjo, Brigitte Jaumard, Napoleão Nepomuceno, Mejdi Kaddour, David Coudert. Dimensioning microwave wireless networks. ICC 2015: IEEE International Conference on Communications, Jun 2015, London, United Kingdom. pp.2803 - 2809, 10.1109/ICC.2015.7248751 • hal-01198461

\section{HAL Id: hal-01198461 \\ https://hal.inria.fr/hal-01198461}

Submitted on 13 Sep 2015

HAL is a multi-disciplinary open access archive for the deposit and dissemination of scientific research documents, whether they are published or not. The documents may come from teaching and research institutions in France or abroad, or from public or private research centers.
L'archive ouverte pluridisciplinaire HAL, est destinée au dépôt et à la diffusion de documents scientifiques de niveau recherche, publiés ou non, émanant des établissements d'enseignement et de recherche français ou étrangers, des laboratoires publics ou privés. 


\title{
Dimensioning Microwave Wireless Networks
}

\author{
Alvinice Kodjo*, Brigitte Jaumard ${ }^{\dagger}$, Napoleão Nepomuceno ${ }^{\ddagger}$, Mejdi Kaddour ${ }^{\S}$ and David Coudert* \\ * Inria, France \& Univ. Nice Sophia Antipolis, CNRS, I3S, UMR 7271, 06900 Sophia Antipolis, France \\ $\dagger$ Department of Computer Science and Software Engineering, Concordia University Montreal (QC) H3G 1M8, Canada \\ $\ddagger$ Graduate Program in Applied Informatics, University of Fortaleza, Fortaleza, Brazil \\ $\S$ Department of Computer Science, University of Oran, Oran 31000, Algeria
}

\begin{abstract}
We aim at dimensioning fixed broadband microwave wireless networks under unreliable channel conditions. As the transport capacity of microwave links is prone to variations due to, e.g., weather conditions, such a dimensioning requires special attention. It can be formulated as the determination of the minimum cost bandwidth assignment of the links in the network for which traffic requirements can be met with high probability, while taking into account that transport link capacities vary depending on channel conditions.

The proposed optimization model represents a major step forward since we consider dynamic routing. Experimental results show that the resulting solutions can save up to $45 \%$ of the bandwidth cost compared to the case where a bandwidth overprovisioning policy is uniformly applied to all links in the network planning. Comparisons with previous work also show that we can solve much larger instances in significantly shorter computing times, with a comparable level of reliability.
\end{abstract}

\section{INTRODUCTION}

Microwave communication describes a traditional transmission technology that is known for its rapid deployment capability, low cost and a flexible application potential. It has been widely deployed in a range of communication contexts including fixed, mobile and private networks. In the context of the steady increase in Internet traffic, fixed broadband wireless networks have become a common alternative to provide private high-speed data connections via microwave [1].

Microwave communication refers to terrestrial point-topoint digital radio communications, usually employing directional antennas in clear line-of-sight (LOS) and operating in licensed frequency bands [2]. Despite recent advances, many questions about how to plan the capacity of wireless microwave networks remain open, especially because of the difficulties in diagnosing channel impairments induced by environmental conditions. Indeed, the dimensioning of microwave wireless networks entails a complex design decision aiming to balance bandwidth-cost efficiency and network reliability, in order to cope with channel fluctuations, due to adaptive modulation in fading channels in order to compensate for, e.g., rain or multipath events.

To support broadband applications, modern microwave systems use quadrature amplitude modulation (QAM). High-level QAM schemes, despite having better bandwidth efficiency, are more susceptible to errors due to channel impairments. To overcome outage events due to fading, modern microwave systems employ adaptive modulation and coding, which have been proven to dramatically improve link performance [3], [4]. In practice, in order to maintain the BER (Bit Error
Rate) performance, this technique involves the variability of the capacity of the links.

Fading phenomena are described in statistical terms and the probability of fades of a particular magnitude can be evaluated through analytical techniques [5]-[7]. Coudert et al [8] suggested identifying a finite set of efficient radio configurations, for which no configuration with a better bandwidth efficiency and a lower SNR (Signal to Noise Ratio) requirement exists. They then associate a discrete probability distribution with these selected configurations, derived either from fading models or power budget calculations.

In the present study, we propose an optimization model in order to dimension fixed broadband wireless microwave networks under unreliable channel conditions, assuming a discrete probability distribution is known for each microwave link and bandwidth. The model determines the minimum cost bandwidth assignment of the links in the network so that a required reliability level of the resulting dimensioning is satisfied, i.e., the selection of bandwidth is made in order to reduce bandwidth costs while ensuring that traffic requirements can be met with high probability. We assume dynamic routing (i.e., routing decisions are made according to channel conditions) to reduce the bandwidth over-provisioning in the network planning.

The paper is organized as follows. In Section II, we discuss the recent related studies on dimensioning microwave networks. The proposed dimensioning model, subject to a bandwidth/modulation probability distribution, is presented in Section III. We then devise a solution of the proposed model in Section IV. It is based on a decomposition technique in order to ensure a scalable solution scheme. Numerical results are described in Section V. Conclusions are drawn in the last section.

\section{RELATED WORK}

We first survey the recent work on the dimensioning of microwave networks (Section II-A), and then summarize the very recent work of [9] that we will use in order to validate the newly proposed dimensioning model (Section II-B).

\section{A. Literature Review}

Computing the probability that a subset of nodes in a probabilistic network is connected is a classical computationally difficult problem [10], [11], even for the case in which the subset of nodes is restricted to a single source-destination pair, 
namely, the two-terminal network reliability problem [12]. But, to the best of our knowledge, Dominiac et al. [13] was the first work to investigate the reliability of fixed broadband wireless networks. The authors, however, assume very strong hypotheses (e.g., single source-destination flow, uncapacitated network, unqualified failures) and apply currently available algorithms for the two-terminal network reliability problem. They only present results for a network with 5 nodes and 7 links.

Recently, the problem of determining the minimum cost bandwidth assignment of a network while guaranteeing a reliability level of the solution was studied in [9], [14], [15]. The authors propose a chance-constrained programming approach in which, if the optimal bandwidth assignment and routing of traffic demands are met, the reliability criterion guarantees that network flows remain feasible with high probability. Under the assumption that links suffer fading independently, they propose reformulations to standard MILP models.

An important point to make in relation to these previous approaches is that they consider static routing. But, in fact, bandwidth assignment and routing decisions take place in different time. Moreover routing decisions vary over time and, therefore, it is possible to reduce the bandwidth utilization by adopting dynamic routing.

\section{B. Budget Constrained Optimization Model}

In [9], the authors proposed as well an alternative budget constrained formulation for which they present a reliability analysis based on different budgets. Instead of minimizing bandwidth costs, their model aims to maximize the reliability of the network while some budget $\mathcal{B}$ is not exceeded.

The network topology is modeled as a digraph $G=(V, L)$, where each node $v \in V$ denotes a radio base station and each link $\ell \in L$ represents a microwave link from $u$ to $v$, with $u, v \in V$. Let $\omega^{+}(v)\left(\omega^{-}(v)\right)$ denote the set of outgoing (incoming) links of $v$. The sets of possible bandwidth values and modulations are denoted by $B$ and $M$, respectively. $B_{\ell} \subseteq B$ denotes the available bandwidth values on link $\ell$. $M_{\ell}^{b} \subseteq M$ denotes the available modulations on $\ell$, subject to the bandwidth selection $b \in B_{\ell}$ on $\ell$. Note that $m \in M$ represents the number of symbols in the $m$-QAM modulation.

Let $\operatorname{COST}_{b}$ be the cost associated with bandwidth choice $b$. Let $\mathrm{CAPA}_{b m}$ be the link capacity for a given bandwidth choice $b$ and a specific compatible modulation $m$. The transport capacity of a link is estimated by the product of the bandwidth value and the bandwidth efficiency of the modulation. Let $\rho_{\ell}^{b m}$ be the probability that link $\ell$, assuming bandwidth choice $b \in B_{\ell}$, is operated with modulation $m \in M_{\ell}^{b}$ or higher (i.e., a modulation that offers a better bandwidth efficiency). Therefore, a feasible routing of traffic demands on link $\ell$ operating with bandwidth selection $b$ and with modulation $m$ is also feasible if the link operates with modulations higher than $m$.

The traffic requirements are modeled by a matrix $D=$ $\left(D_{s d}\right)$, where $D_{s d}$ denotes the amount of traffic from $s$ to $d$. Let $\mathcal{S D}=\left\{(s, d) \in V^{2}: D_{s d}>0\right\}$. Let $a_{\ell, b m}$ be the binary decision variable indicating whether the bandwidth/modulation pair $(b, m)$ is assigned or not to link $\ell \in L$. Flow variables $\varphi_{\ell}^{s d}$ denote the fraction of $D_{s d}$ that is routed on link $\ell \in L$.

$$
\max \sum_{\ell \in L} \sum_{b \in B_{\ell}} \sum_{m \in M_{\ell}^{b}} \log \left(\rho_{\ell}^{b m}\right) a_{\ell, b m}
$$

subject to:

$$
\begin{aligned}
& \sum_{\ell \in \omega^{-}(v)} \varphi_{\ell}^{s d}-\sum_{\ell \in \omega^{+}(v)} \varphi_{\ell}^{s d}= \begin{cases}-D_{s d}, & \text { if } v=s, \\
D_{s d}, & \text { if } v=d, \\
0, & \text { otherwise } \\
v \in V,(s, d) \in \mathcal{S D}\end{cases} \\
& \sum_{(s, d) \in \mathcal{S D}} \varphi_{\ell}^{s d} \leq \sum_{b \in B_{\ell}} \sum_{m \in M_{\ell}^{b}}{ }_{\operatorname{CAPA}_{b m} a_{\ell, b m} \quad}^{\ell \in L} \\
& \sum_{b \in B_{\ell}} \sum_{m \in M_{\ell}^{b}} a_{\ell, b m} \leq 1 \\
& \sum_{\ell \in L} \sum_{b \in B_{\ell}} \sum_{m \in M_{\ell}^{b}} \operatorname{CosT}_{b} a_{\ell, b m} \leq \mathcal{B} \\
& \varphi_{\ell}^{s d} \geq 0 \quad \ell \in L \\
& a_{\ell, b m} \in\{0,1\} \quad \ell \in L, b \in B_{\ell}, m \in M_{\ell}^{b} .
\end{aligned}
$$

Objective function (1) represents the linearization, using a logarithmic function, of the network reliability based on the assigned bandwidth values and the independence of the link outages. Flow conservation constraints (2) determine the routes that fulfill the traffic requirement for each demand. Constraints (3) ensure that bandwidth assignment is appropriate in order to support the overall requested traffic demand. Constraints (4) guarantee that a unique pair $(b, m)$ is assigned to each link. Finally (5) enforces the cost of the solution not to exceed the budget value $\mathcal{B}$.

\section{Network Model}

\section{A. Definitions and Assumptions}

We will re-use in this section the same notations and definitions related to network topology model, radio configurations and traffic demands, which we introduced in Section II-B. In addition, let $M_{\ell} \subseteq M$ be the set of all available modulations on link $\ell$ regardless of the bandwidth. Let $\mathrm{BM}_{\ell}$ be the set of all possible bandwidth/modulation pairs on $\ell$. Note however that, in the new proposed model, the definition of the probability distribution associated with the pairs in $\mathrm{BM}_{\ell}$ is slightly different. Instead of using the cumulative probability distribution as in [9], we use a set of modulation distributions:

$$
\sum_{m:(b, m) \in \mathrm{BM}_{\ell}} \pi_{\ell, b m}=1 \quad \ell \in L, b \in B_{\ell},
$$

where $\pi_{\ell, b m}$ denotes the probability that modulation $m$ is used together with bandwidth $b$ on link $\ell$.

Let us now define the notion of configuration of a network: a configuration $c$ corresponds to a network dimensioning (i.e., bandwidth selection for each link) and, given the dimensioning, a radio selection (i.e., modulation for each link). Formally, 
the binary parameters $a_{\ell, b}^{c}$ and $a_{\ell, m}^{c}$ denote whether link $\ell$ uses bandwidth $b$ and modulation $m$, respectively, within the configuration $c$. Parameter $a_{\ell, b m}^{c}$ is set to 1 for link $\ell$ if the corresponding bandwidth and modulation parameters are also equal to 1 in configuration $c$, i.e., $a_{\ell, b m}^{c}=a_{\ell, b}^{c} a_{\ell, m}^{c}$. Note that only one bandwidth and one modulation value can be associated with each link in a given configuration.

Due to the independence between the probability distribution for each link, the probability $p^{c}$ of a configuration $c$ can be written as:

$$
p^{c}=\prod_{\ell \in L} \sum_{(b, m) \in \mathrm{BM}_{\ell}} \pi_{\ell, b m} a_{\ell, b m}^{c} \quad c \in C
$$

where $C$ represents the set of all possible configurations.

Note that it is possible that not all traffic demands are completely satisfied by the configurations of $C$, due to the capacity constraints vs. the traffic demand. For that reason, we introduce the variable $\delta_{s d}^{c}$ that is equal to the amount of unsatisfied demand from source node $s$ to destination node $d$.

\section{B. Optimization Model}

The objective is to assign the bandwidth values on the links of $L$ in order to minimize the bandwidth cost for provisioning the granted requests. However, due to the capacity constraints, and the strong conditions imposed by the modulation probability distributions, it may happen that the model has no solution. Then, in order to ensure that the optimization model has always a solution, we minimize the bandwidth cost only after the minimization of the amount of unsatisfied demands and of some possible unfeasibility with respect to the probabilities of the modulation distributions. Variables of the proposed model are:

- $x_{c}=1$ if configuration $c$ is selected as a relevant network dimensioning configuration, 0 otherwise

- $z_{\ell, b}=1$ if bandwidth $b$ is used on link $\ell$ in all selected configurations, 0 otherwise.

- $z_{\ell}=$ bandwidth cost of link $\ell$

- $y_{\ell, b m}=$ amount of unfeasibility with respect to the discrete probability modulation distribution for a given link $\ell$ and $(b, m) \in \mathrm{BM}_{\ell}$.

In preliminary experiments, we observed that the model may contain numerous configurations with an extremely small probability. Also we decided to search for an optimal solution made of configurations with meaningful probabilities. As a consequence, the probability that some link $\ell$ uses configuration $(b, m)$ in the solution does not necessarily match the given probability $\pi_{\ell, b m}$. We then introduced the variable $y_{\ell, b m}$ in order to always guarantee a solution for our optimization model. Yet, we ensure that the solution provides a satisfactory grade of service to the network operator by enforcing that $\sum_{c \in C} p^{c}$ is at least $p_{\min }$, with $p_{\min }$ quite close to 1 . In the sequel, we refer to $\sum_{c \in C} p^{c} x_{c}$ as the reliability of the microwave network dimensioning.
Our optimization model can now be stated as follows:

$$
\begin{aligned}
& \min \sum_{\ell \in L} z_{\ell}+\text { PENAL }_{1} \sum_{(s, d) \in \mathcal{S} \mathcal{D}} \sum_{c \in C} \delta_{s d}^{c} p^{c} x_{c} \\
& +\operatorname{PENAL}_{2} \sum_{\ell \in L} \sum_{(b, m) \in \mathrm{BM}_{\ell}} y_{\ell, b m} \\
& \sum_{c \in C} p^{c} x_{c} \geq p_{\min } \\
& \sum_{c \in C} a_{\ell, b m}^{c} p^{c} x_{c}+\quad y_{\ell, b m}=z_{\ell, b} \pi_{\ell, b m} \quad \ell \in L, \\
& m \in M_{\ell}, b \in B_{\ell}:(b, m) \in \mathrm{BM}_{\ell} \\
& \sum_{b \in B_{\ell}} z_{\ell, b}=1 \quad \ell \in L \\
& \operatorname{COST}_{b} z_{\ell, b} \leq z_{\ell} \quad \ell \in L, b \in B_{\ell} \\
& z_{\ell, b} \in\{0,1\} \quad \ell \in L, b \in B_{\ell} \\
& z_{\ell} \geq 0 \quad \ell \in L \\
& x_{c} \in\{0,1\} \quad c \in C \\
& y_{\ell, b m} \in[0,1] \quad \ell \in L,(b, m) \in \mathrm{BM}_{\ell} \text {. }
\end{aligned}
$$

PENAL $_{1}$ and PENAL 2 are positive constants prioritizing the minimization of unsatisfied demand and probability distribution unfeasibility, respectively. The last unfeasibility is determined using variables $\left(y_{\ell, b m}\right)$, for each link, in constraint (12) according to the selected configurations $\left(x_{c}\right)$ and the assigned bandwidth $b\left(z_{\ell, b}\right)$. Constraints (14) determine the bandwidth cost value for a given link. Note that we do not need to explicitly impose variables $z_{\ell}$ to be integer in (16) as those integrality constraints are implicitly entailed by the other constraints.

Observe that the number of constraints is in the order of $\mathrm{O}(|L| \times|\mathrm{BM}|)$. This remains reasonable as the number of elements in $\mathrm{BM}$ is quite limited in practice.

\section{An Illustrative Example}

Consider a small microwave network with three links : $\ell_{1}, \ell_{2}, \ell_{3}$. We use the following bandwidth and modulation values:

$$
\text { - } \mathrm{B}=\left\{b_{1}=7 \mathrm{MHz}, b_{2}=14 \mathrm{MHz}\right\}
$$$$
\text { - } \mathrm{M}=\left\{m_{1}=\text { QPSK, } m_{2}=16 \mathrm{QAM}\right\}
$$

Thus,

$$
\begin{aligned}
\mathrm{BM}=\{(7 \mathrm{MHz}, & \mathrm{QPSK}),(7 \mathrm{MHz}, 16 \mathrm{QAM}), \\
& (14 \mathrm{MHz}, \mathrm{QPSK}),(14 \mathrm{MHz}, 16 \mathrm{QAM})\} .
\end{aligned}
$$

The discrete probability distributions of the bandwidth/modulation of each link are given in Table I. Note that there are 64 possible network configurations. Table II shows only the selected network configurations $z_{\ell_{1}, b_{1}}=z_{\ell_{2}, b_{1}}=$ $z_{\ell_{3}, b_{1}}=1$.

Assume that each link carries a traffic demand which can be satisfied by any combination in BM. Clearly, a solution that assigns $b_{1}$ to each link will be the less-costly for the network operator. Furthermore, if all eight configurations are selected, 
TABLE I

MODULATION DISCRETE PROBABILITY DISTRIBUTIONS

\begin{tabular}{|c|c|c|c|c|}
\hline Links & $\pi_{\ell, b_{1} m_{1}}$ & $\pi_{\ell, b_{1} m_{2}}$ & $\pi_{\ell, b_{2} m_{1}}$ & $\pi_{\ell, b_{2} m_{2}}$ \\
\hline$\ell_{1}$ & 0.1 & 0.9 & 0.2 & 0.8 \\
$\ell_{2}$ & 0.2 & 0.8 & 0.3 & 0.7 \\
$\ell_{3}$ & 0.1 & 0.9 & 0.2 & 0.8 \\
\hline
\end{tabular}

TABLE II

CONFIGURATIONS FOR $z_{\ell_{1}, b_{1}}=z_{\ell_{2}, b_{1}}=z_{\ell_{3}, b_{1}}=1$

\begin{tabular}{|c|c|c|c|c|c|c|c|c|c|}
\hline \multicolumn{2}{|c|}{$L / \mathrm{BM}$} & $c_{1}$ & $c_{2}$ & $c_{3}$ & $c_{4}$ & $c_{5}$ & $c_{6}$ & $c_{7}$ & $c_{8}$ \\
\hline \multirow{4}{*}{$\ell_{1}$} & $\left(b_{1}, m_{1}\right)$ & 1 & 1 & 1 & 1 & 0 & 0 & 0 & 0 \\
\hline & $\left(b_{1}, m_{2}\right)$ & 0 & 0 & 0 & 0 & 1 & 1 & 1 & 1 \\
\hline & $\left(b_{2}, m_{1}\right)$ & 0 & 0 & 0 & 0 & 0 & 0 & 0 & 0 \\
\hline & $\left(b_{2}, m_{2}\right)$ & 0 & 0 & 0 & 0 & 0 & 0 & 0 & 0 \\
\hline \multirow{4}{*}{$\ell_{2}$} & $\left(b_{1}, m_{1}\right)$ & 1 & 1 & 0 & 0 & 1 & 1 & 0 & 0 \\
\hline & $\left(b_{1}, m_{2}\right)$ & 0 & 0 & 1 & 1 & 0 & 0 & 1 & 1 \\
\hline & $\left(b_{2}, m_{1}\right)$ & 0 & 0 & 0 & 0 & 0 & 0 & 0 & 0 \\
\hline & $\left(b_{2}, m_{2}\right)$ & 0 & 0 & 0 & 0 & 0 & 0 & 0 & 0 \\
\hline \multirow{4}{*}{$\ell_{3}$} & $\left(b_{1}, m_{1}\right)$ & 1 & 0 & 1 & 0 & 1 & 0 & 1 & 0 \\
\hline & $\left(b_{1}, m_{2}\right)$ & 0 & 1 & 0 & 1 & 0 & 1 & 0 & 1 \\
\hline & $\left(b_{2}, m_{1}\right)$ & 0 & 0 & 0 & 0 & 0 & 0 & 0 & 0 \\
\hline & $\left(b_{2}, m_{2}\right)$ & 0 & 0 & 0 & 0 & 0 & 0 & 0 & 0 \\
\hline & $p^{c}$ & 0.002 & 0.018 & 0.008 & 0.072 & 0.018 & 0.162 & 0.072 & 0.648 \\
\hline
\end{tabular}

the reliability will be equal to 1 and the terms multiplied by PENAL $_{2}$ in (10) will be equal to zero. Hence, the objective function is minimized. However, if a minimum reliability level of 0.8 is sufficient for the network operator, the solution consisting in the three configurations $c_{6}, c_{7}$ and $c_{8}$ would satisfy all the requirements with a reliability level equals to $0.882(0.162+0.072+0.648)$

Now, if we consider larger problem instances (see Section $\mathrm{V})$, we cannot enumerate explicitly all possible configurations, hence we describe in the next section how to solve the model (10) - (18) using an implicit enumeration of all configurations, thanks to the column generation method. In the numerical experiments, we will find a compromise between the value of $p_{\text {min }}$, i.e., an acceptable reliability, and the generation of meaningful configurations, i.e., configurations with a probability value that is meaningful.

\section{Solution of the Proposed Model}

\section{A. Outline}

A straightforward solution scheme for the model (10)-(18) is to enumerate all possible configurations: this is intractable even with moderate-size instances. In this section, we describe a solution process based on the Column Generation method that splits the original problem into two subproblems: the Restricted Master Problem (RMP) and the Pricing Problem. The RMP is a linear relaxation of the original problem where only a limited subset of configurations is considered, while the pricing generates, one at a time, the configurations that have the potential to improve the objective function of the current RMP, i.e., to find RMP variables (or, equivalently, RMP columns) with negative reduced costs, see, e.g., [16] for more details. The column generation method is to alternate the solution of these problems until the optimality condition is satisfied: no more configurations (i.e., RMP variables/columns) with a negative reduced cost can be derived when solving the pricing problem.

\section{B. Pricing Problem}

In this section, we omit the index $c$ to alleviate the notations.

The objective of the pricing problem consists in minimizing the reduced cost of variables $x_{c}$. From (10)-(12), we have:

$$
\begin{aligned}
\min \overline{\mathrm{COST}}=\prod_{\ell \in L} p_{\ell}\left(\operatorname{PENAL}_{1} \sum_{(s, d) \in \mathcal{S} \mathcal{D}} \delta_{s d}-u^{(11)}\right. \\
\left.-\sum_{\ell \in L} \sum_{(b, m) \in \mathrm{BM}_{\ell}} u_{\ell, b m}^{(12)} a_{\ell, b m}\right)
\end{aligned}
$$

where $u^{(11)}(\geq 0)$ and $u_{\ell, b m}^{(12)}(\lessgtr 0)$ are dual values corresponding to constraints (11) and (12), respectively. $p_{\ell}$ represents the probability that the link $\ell$ uses the affected bandwidth/modulation in the configuration under construction. The set of constraints of the pricing problem is as follows:

$$
\begin{aligned}
& p_{\ell}=\sum_{(b, m) \in \mathrm{BM}_{\ell}} \pi_{\ell, b m} a_{\ell, b m} \quad \ell \in L \\
& \sum_{\ell \in \omega^{-}(v)} \varphi_{\ell}^{s d}-\sum_{\ell \in \omega^{+}(v)} \varphi_{\ell}^{s d}= \begin{cases}-D_{s d}+\delta_{s d} & v=s \\
D_{s d}-\delta_{s d} & v=d \\
0 & \text { otherwise }\end{cases}
\end{aligned}
$$

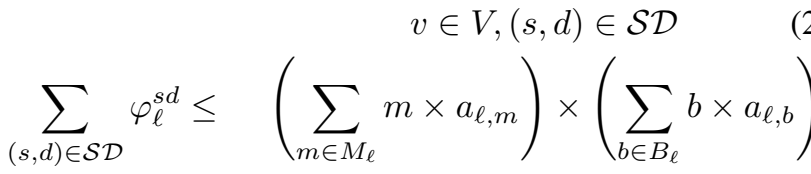

$$
\begin{aligned}
& \ell \in L \\
& \sum_{m \in M_{\ell}} a_{\ell, m}=1 \quad \ell \in L \\
& \sum_{b \in B_{\ell}} a_{\ell, b}=1 \quad \ell \in L \\
& \sum_{(b, m) \in \mathrm{BM}_{\ell}} a_{\ell, b m}=1 \quad \ell \in L \\
& a_{\ell, b m}=a_{\ell, m} a_{\ell, b} \\
& \ell \in L, m \in M, b \in B:(b, m) \in \mathrm{BM}_{\ell} \\
& \varphi_{\ell}^{s d} \geq 0 \quad \ell \in L,(s, d) \in \mathcal{S D} \\
& a_{\ell, b m} \in\{0,1\} \ell \in L, \\
& m \in M, b \in B:(b, m) \in \mathrm{BM}_{\ell} \\
& a_{\ell, b} \in\{0,1\} \quad \ell \in L, b \in B_{\ell} \\
& a_{\ell, m} \in\{0,1\} \quad \ell \in L, m \in M_{\ell} \\
& \delta_{s d} \geq 0 \quad(s, d) \in \mathcal{S D} \\
& 0 \leq p_{\ell} \leq 1 \quad \ell \in L
\end{aligned}
$$

Constraints (21) enforce the flow conservation for a given selection of the bandwidth/modulation assignment to each link as suggested by the values of the dual variables, while the flow on each link is limited (Constraints (22)) by the transport capacity defined by the selected pair of bandwidth/modulation on each link, in the configuration under construction. 
Solving the pricing problem presents two challenges. The first one is due to the presence of the quadratic term $a_{\ell, m} a_{\ell, b}$ : it can be easily overcome with the following linearization:

$$
\begin{aligned}
& a_{\ell, b m} \geq a_{\ell, m}+a_{\ell, b}-1 \\
& a_{\ell, m} \geq a_{\ell, b m} \\
& a_{\ell, b} \geq a_{\ell, b m}
\end{aligned}
$$

The second challenge, that is the product of $p_{\ell}$ variables, is more difficult to address as it corresponds to a non convex function. In the sequel, we propose a heuristic approach to solve the pricing problem, which takes care of this nonlinear non convex term.

\section{Solution Process}

In the column generation process, we only add to the current RMP those configurations generated by the pricing problem, for which the objective value (linear programming reduced cost) of the pricing problem is negative. To overcome the difficulty of solving the pricing problem due to the presence of the non convex term, we introduce a slight transformation. We know that the term $\prod_{\ell \in L} p_{\ell}$ is always positive and with a rather small value as it is the product of probabilities; consequently, it has little impact on the sign of the optimal value of the reduced cost. The idea is therefore to first solve the pricing problem with the omission of the first non-linear term in the objective. In this way, we are guaranteed to solve the pricing problem while generating all useful configurations that can improve the RMP objective function. Moreover, we consider only the configurations that satisfy all the traffic requirements. After a first configuration $c$ with a negative reduced cost has been found through this simplified pricing model, we use a local search heuristic in order to generate other valid configurations with a negative reduced cost in the neighbourhood of the first configuration, and which be also added to the RMP. The aim is to speed up the solution of the linear relaxation of the master problem. These configurations are derived from $c$ by modifying the modulation of some randomly selected links of configuration $c$ : if their reduced cost is negative, they are retained, otherwise they are forgotten.

The first step of the solution process, (upper rectangle in the flowchart of Figure 1), consists then to solve iteratively the RMP and the modified pricing problem until no more configuration with a negative reduced cost can be found. The first step is followed by an ILP resolution of the last RMP that results in a set of network configurations with a minimum bandwidth cost. Notice that the proposed solution process, because of the use of the modified pricing problem, and the ILP solution of the last RMP (instead of a branchand-price method, see, e.g., [17]) is a heuristic method. In order to increase the dimensioning reliability, we apply a postoptimization procedure. It first chooses the configuration, say $c_{1}$, with the highest probability among those that were selected in the ILP solution of the last RMP. Therein, the bandwidth of each link is already assigned. Then, we derive new configurations by modifying the modulation of some randomly chosen links of $c_{1}$. We next use the simplified pricing problem in order to verify the existence of a routing solution for these configurations, if they have a negative reduced cost. If a routing exists, then the configurations are added to the current RMP. Computational experiments showed that such a postprocessing was useful to increase the dimensioning reliability without modifying the bandwidth assignment of $c_{1}$.

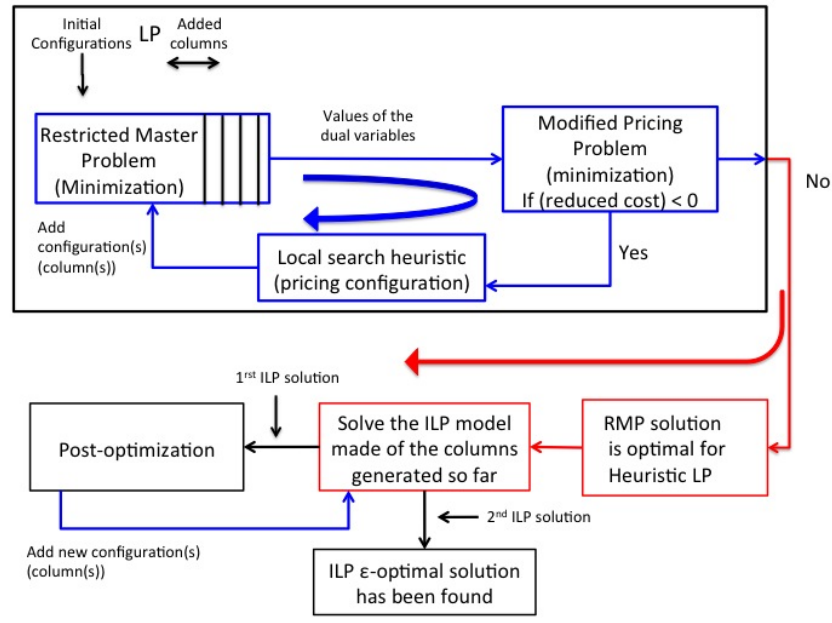

Fig. 1. ILP and Column Generation Solution Process

\section{Initial Solution of the RMP}

Solving the RMP model requires a satisfactory value for $p_{\min }$, that is the desired reliability level predefined by the network operator, and a subset of initial configurations such that (11) is satisfied. However, it can be difficult for large instances (i.e., slow convergence) to reach a feasible solution such that (11) is satisfied depending on the value of $p_{\min }$. We overcome this issue by solving a modified version of model (10)-(18), which is defined as follows. We first modify the objective function (10), and replace it by the following one:

$$
\max \sum_{c \in C} p^{c} x_{c}-\operatorname{PENAL}_{1} \sum_{\left(v_{s}, v_{d}\right) \in \mathcal{S} \mathcal{D}} \sum_{c \in C} \delta_{s d}^{c} p^{c} x_{c}
$$

in order to first generate configurations which maximizes $\sum_{c \in C} p^{c} x_{c}$ in order to quickly satisfy (11), and check that the selected value for $p_{\min }$ is not too difficult to reach in practice. We keep all the constraints except for (11).

The objective function of the corresponding pricing problem needs to be modified accordingly and is written as follows:

$$
\begin{aligned}
\max \overline{\operatorname{COST}^{\prime}=} & \left(1-\operatorname{PENAL}_{1} \sum_{(s, d) \in \mathcal{S} \mathcal{D}} \delta_{s d}\right) \prod_{\ell \in L} p_{\ell} \\
& -\prod_{\ell \in L} p_{\ell} \sum_{\ell \in L} \sum_{(b, m) \in \mathrm{BM}_{\ell}} u_{\ell, b m}^{(12)} a_{\ell, b m}
\end{aligned}
$$

while the set of constraints is not modified.

Again the non-linearity in the objective of the pricing problem is overcome thanks to the heuristic approach described in the previous section. Note that, as we now solve 
a maximization problem, a new configuration will be added to the initial RMP if the corresponding reduced cost $\overline{\mathrm{COST}^{\prime}}$ is positive.

When no more improving configurations can be generated, then we switch to the solution process described in the flowchart of Figure 1.

\section{NumericAl RESUlts}

\section{A. Setting of the Experiments}

We now describe the set of experiments we conducted in order to evaluate the quality of the solutions output by our optimization model. We consider a set of realistic network instances taken from SNDlib [18] with the traffic demands rescaled as in [9]: Atlanta (15 nodes, 44 links), Polska (12 nodes, 36 links), France ( 25 nodes, 90 links) and Germany50 (50 nodes, 176 links). We use the following parameters:

- $\mathrm{B}=\left\{b_{1}=7 \mathrm{MHz}, b_{2}=14 \mathrm{MHz}, b_{3}=28 \mathrm{MHz}\right\}$

- $\mathbf{M}=\left\{m_{1}=16\right.$-QAM coded, $m_{2}=16$-QAM uncoded

$m_{3}=64-\mathrm{QAM}$ coded, $m_{4}=64-\mathrm{QAM}$ uncoded

$m_{5}=256$-QAM coded, $m_{6}=256$-QAM uncoded $\}$

This leads to $18(b, m)$ pairs. Note that the network instances as well as the radio parameters are identical to those used in [9]. We set a normalized monetary cost of $\$ 1,000$ per $1 \mathrm{MHz}$ of bandwidth. Besides, based on the Vigants-Barnett radio fading model [6], the probability distributions of the considered modulations are such that $\forall \ell \in L, \forall b \in B, \pi_{\ell, b m_{6}}$ is in the order of 0.999 while all other modulations are around $10^{-5}$. Note also that the solution process of the column generation subprocess is limited to two hours. Other parameters are as follows: PENAL $_{1}=40,000 ;$ PENAL $_{2}=50 ; p_{\min }=0.9$.

The high variability of the probability distributions described above and the independence between link probabilities produce a tremendous number of possible configurations with very low probability value. In order to prevent generating such meaningless configurations, e.g., with probability of the order of $10^{-75}$, we requested that: $p_{\ell} \geq 0.1$ for $\ell \in L$ in the pricing problem.

TABLE III

CG RESULTS

\begin{tabular}{|l|c|c|c|c|}
\cline { 2 - 5 } \multicolumn{1}{c|}{} & Polska & Atlanta & France & Germany50 \\
\hline \# Total conf. & 606 & 635 & 629 & 454 \\
\hline \# Used conf. & 78 & 16 & 102 & 17 \\
\hline
\end{tabular}

Table III shows that the total number of configurations generated by our solution process represents only a tiny fraction of the overall number of possible configurations, i.e., $\prod_{\ell \in L}\left|\mathrm{BM}_{\ell}\right|$. That means that our solution process focuses primarily on the most significant configurations in terms of probability and demand satisfaction. Also, we can observe that the best solutions in terms of cost will consider only a limited number of configurations while reaching the desired dimensioning reliability level. Note that, in practice, generated configurations satisfy all traffic demands, i.e., $\delta_{s d}=0$ for all $(s, d) \in \mathcal{S D}$.

\section{B. Solution quality}

We highlight here the quality of the solutions obtained by our optimization model and associated solution process. Fig. 2 shows, for each network topology, the cost saving that can be achieved by the network operator compared to the worst case where the highest cost bandwidth is installed on every link. We observe a significant cost saving ranging from $33 \%$ to $45 \%$, while achieving a high dimensioning reliability. As the saving increases with the network size, one can notice some decrease for the dimensioning reliability. This is due in part to the additional constraint on $p_{\ell}\left(p_{\ell} \geq 0.1\right)$ but mostly to the fact that it is much harder to maintain the same dimensioning reliability because the configuration probabilities decrease by several orders of magnitude when considering more links in a network.

The quality of our method can also be evaluated through the reliability gaps between our solutions and the worst case, as shown in Fig. 3. We can see that the dimensioning reliability is only marginaly compromised even if the total bandwidth is minimized.

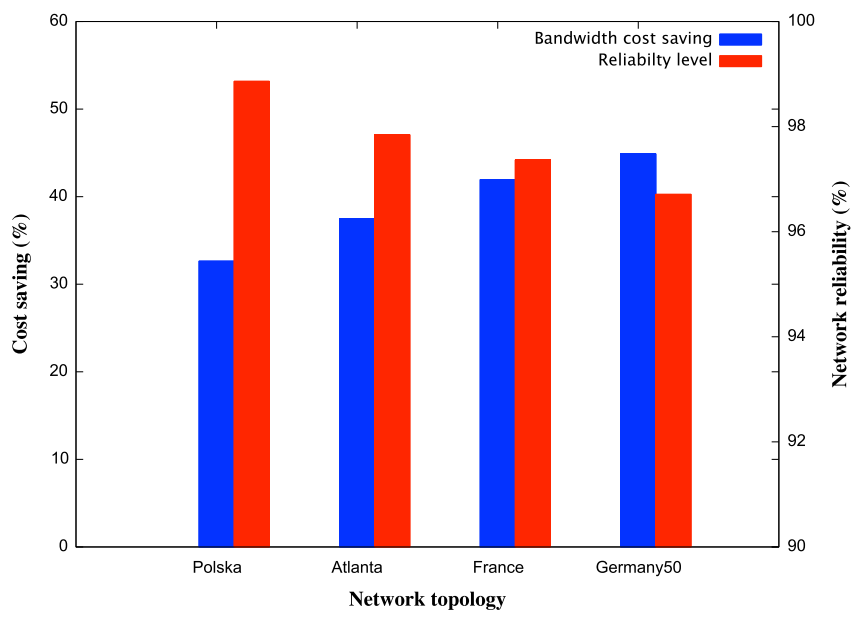

Fig. 2. Cost saving vs. the worst case

\section{Validation of the Results: Comparison with those of [9]}

Another way to evaluate our results is to compare them against those reported by Claßen et al. [9]. First, it is important to recall that the problem addressed in [9] is slightly different from ours. As exposed in Section II-B, the methodology followed in [9] is to maximize the reliability for a fixed budget $\mathcal{B}$ (Constraints (1) to (5)). This last problem is solved multiple times for decreasing budgets $\mathcal{B}$ until no solution can be found. While this approach allows finding a good compromise between cost and reliability, its drawback is its very long required computational times in order to find the minimum cost solution.

Using this budget-constrained formulation, the resulting solutions provide $36 \%, 39 \%$ and $40 \%$ of cost savings for Polska, Atlanta and France networks, respectively. Our results depicted in Fig. 2 are inferior by no more than 3\% for Polska 


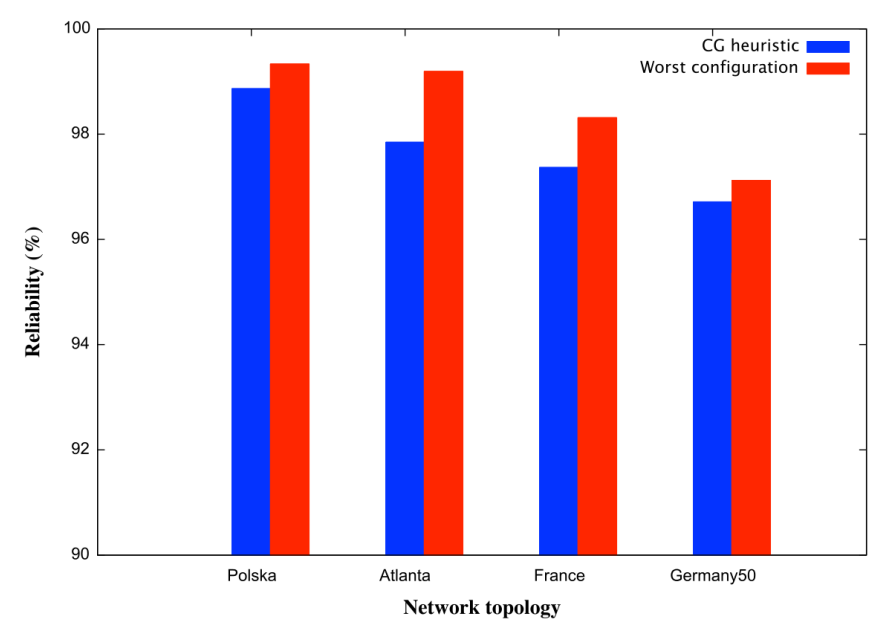

Fig. 3. Reliability vs. the worst case

TABLE IV

RESOLUTION TIME (MINUTES)

\begin{tabular}{|c|c|c|c|c|}
\cline { 2 - 5 } \multicolumn{1}{c|}{} & Polska & Atlanta & France & Germany50 \\
\hline CG heuristic & 7 & 32 & $131^{*}$ & $148^{*}$ \\
\hline $\begin{array}{c}\text { Chance- } \\
\text { constrained }\end{array}$ & 21 & 120 & nf & - \\
\hline
\end{tabular}

*: CG process is stopped after 2 hours.

nf: no feasible solution was reported, - : not considered.

and Atlanta while our method performs better on the France instance as no solution has been found with the same cost in their case. Also, the dimensioning reliabilities are very close as the gap is less than $1 \%$ for these three topologies.

In summary, chance-constraint method provides better solutions on small network instances while our approach based on column generation scales much better. This is explained by the ILP formulation of the chance-constrained method that is not scalable. We believe that obtaining feasible solutions with this approach in a reasonable amount of time is very unlikely for large instances, such as Germany50. Table IV summarizes a comparison of the computation times required in both cases, in to obtain the solutions of similar cost. Not only our approach is the fastest one, but it also provides good results for large instances in a relatively short amount of time.

\section{CONCLUSION}

In this paper, we have proposed a new linear programming formulation, using column generation, for the dimensioning of fixed broadband microwave wireless networks under unreliable channel conditions. Furthermore, we have shown how to overcome the difficulty raised by the non-linear (and non convex) objective of the pricing problem by using a heuristic. We have assessed the potential of our approach on large scale instances. In particular, we were able to solve instances that were not reachable by other methods.

As future work, we plan to improve the heuristic used to solve the pricing problem in order to increase the reliability of the solutions, and eventually find solutions with smaller cost. We would also like to propose a fixed budget formulation in order to build the Pareto front of the solution space. Moreover, we will investigate on the correlations of the links fades that are due to environmental (weather) conditions.

\section{ACKNOWLEDGMENT}

B. Jaumard was supported by a Concordia University Research Chair (Tier I) and by an NSERC (Natural Sciences and Engineering Research Council of Canada) grant. M. Kaddour was supported by the TASSILI research program 11MDU839 between the University of Oran (Algeria) and the University of Nice (France). D. Coudert and A. Kodjo were supported by ANR program "Investments for the Future" under reference ANR-11-LABX-0031-01. N. Nepomuceno was supported by FUNCAP/CNRS INC-0083-00047.01.00/13.

\section{REFERENCES}

[1] H. Anderson, Fixed Broadband Wireless System Design. John Wiley \& Sons, 2003.

[2] H. Lehpamer, Microwave transmission networks: planning, design, and deployment. McGraw-Hill, 2010.

[3] A. Goldsmith and S.-G. Chua, "Variable-rate variable-power MQAM for fading channels," IEEE Transactions on Communications, vol. 45, pp. $1218-1230,1997$.

[4] — - "Adaptive coded modulation for fading channels," IEEE Transactions on Communications, vol. 46, no. 5, pp. 595-602, 1998.

[5] W. Barnett, "Multipath propagation at 4, 6 and $11 \mathrm{GHz}$," Bell System Technical Journal, vol. 51, no. 2, pp. 311-361, 1972.

[6] A. Vigants, "Space-diversity engineering," Bell System Technical Journal, vol. 54, no. 1, pp. 103-142, 1975.

[7] R. K. Crane, Electromagnetic wave propagation through rain. John Wiley \& Sons, 1996.

[8] D. Coudert, N. Nepomuceno, and H. Rivano, "Power-efficient radio configuration in fixed broadband wireless networks," Computer Communications, vol. 33, no. 8, pp. 898-906, 2010.

[9] G. Classen, D. Coudert, A. M. Koster, and N. Nepomuceno, "Chanceconstrained optimization of reliable fixed broadband wireless networks," INFORMS Journal of Computing, vol. xx, no. xx, p. to appear, 2014

[10] M. Ball, "Computational complexity of network reliability analysis: An overview," IEEE Transactions on Reliability, vol. 35, no. 3, pp. 230-239, 1986.

[11] P. Hansen, B. Jaumard, and G.-B. Douanya-Nguetse, "Best second order bounds for two-terminal network reliability with dependent edge failures," Discrete Applied Mathematics, vol. 9697, p. 375393, October 1999.

[12] T. Brecht and C. Colbourn, "Lower bounds on two-terminal network reliability," Discrete Applied Mathematics, vol. 21, no. 3, pp. 185-198, 1988.

[13] S. Dominiak, N. Bayer, J. Habermann, V. Rakocevic, and B. Xu, "Reliability analysis of IEEE 802.16 mesh networks," in 2nd IEEE/IFIP International Workshop on Broadband Convergence Networks, BcN 2007, 2007, pp. 1-12.

[14] G. Claßen, D. Coudert, A. M. C. A. Koster, and N. Nepomuceno, "Bandwidth assignment for reliable fixed broadband wireless networks," in 12th IEEE International Symposium on a World of Wireless Mobile and Multimedia Networks (WoWMoM 2011), 2011, pp. 1-6.

[15] — , "A chance-constrained model and cutting planes for fixed broadband wireless networks," in Network optimization, ser. Lectures Notes in Computer Science, 2011, vol. 6701, pp. $37-42$.

[16] V. Chvatal, Linear Programming. Freeman, 1983.

[17] C. Barnhart, E. Johnson, G. Nemhauser, M. Savelsbergh, and P. Vance, "Branch-and-price: Column generation for solving huge integer programs," Operations Research, vol. 46, no. 3, pp. 316-329, 1998.

[18] S. Orlowski, M. Pióro, A. Tomaszewski, and R. Wessäly, "SNDlib 1.0-Survivable Network Design Library," in Proceedings of the 3rd International Network Optimization Conference (INOC 2007), Spa, Belgium, April 2007, pp. 276-286. 